\title{
Exact Chiral Fermions and Finite Density on Lattice
}

\section{Debasish Banerjee}

Department of Theoretical Physics, Tata Institute of Fundamental Research, Homi Bhabha Road, Mumbai 400005, India

E-mail:debasishetheory.tifr.res.in

\section{Rajiv V. Gavai*†}

Department of Theoretical Physics, Tata Institute of Fundamental Research, Homi Bhabha Road, Mumbai 400005, India

E-mail:gavai@tifr.res.in

\section{Sayantan Sharma *}

Department of Theoretical Physics, Tata Institute of Fundamental Research, Homi Bhabha Road, Mumbai 400005, India

E-mail:ssharma@theory.tifr.res.in

Any $\mu^{2}$-divergence is shown analytically to be absent for a class of actions for Overlap and Domain Wall Fermions with nonzero chemical potential. All such actions are, however, shown to violate the chiral invariance. While the parameter $M$ of these actions can be shown to be irrelevant in the continuum limit, as expected, it is shown numerically that the continuum limit can be reached with relatively coarser lattices for $1.5 \leq M \leq 1.6$.

The XXVI International Symposium on Lattice Field Theory

July 14-192008

Williamsburg, Virginia, USA

\footnotetext{
* Speaker.

${ }^{\dagger}$ Supported by the Indian Lattice Gauge Theory Initiative project of TIFR, Mumbai, India.

${ }^{\ddagger}$ Supported by the Shyamaprasad Mukherjee Fellowship of CSIR, India.
} 


\section{Introduction.}

Quantum Chromo Dynamics (QCD) with massless quarks of $N_{f}$ flavours has $S U\left(N_{f}\right) \times S U\left(N_{f}\right)$ chiral symmetry which is dynamically broken at low temperatures by the vacuum. Since the $u$ and $d$ quarks have almost degenerate mass which in turn is much smaller than the scale of QCD, and the $s$ quark is only moderately heavy, approximate chiral symmetry is a reasonable assumption for our world, i.e., QCD with $2+1$ flavours of dynamical quarks. Rich phenomenological studies have demonstrated the utility, and consistency of such an assumption. Pions and Kaons are thus widely regarded as the Goldstone bosons resulting from the dynamical symmetry breaking by vacuum. It is thus a natural consequence that the finite temperature transition in our world is also widely accepted to be governed by chiral symmetry. It is therefore desirable to have the chiral invariance in any formulation which aims at studying the thermodynamics of QCD. Let us cite below two important physics aspects where it is even necessary to have exact chiral invariance.

Lattice QCD has clearly been the method of choice for reliable non-perturbative studies in general, and finite temperature/density investigations in particular. As is well known, the Fermion doubling problem makes it difficult to demand the same chiral invariance as in continuum QCD. The original solution to this problem, namely, the Wilson Fermions, breaks all chiral symmetries. Since the staggered Fermions do have an exact chiral symmetry on the lattice, albeit at the cost of breaking of flavour and spin symmetries, they have dominated the area of nonzero temperatures and densities.

As presented [1] in Lattice 2006 by one of us, the hadronic screening lengths illustrate their deficiency in the pionic screening length whereas the Overlap Fermions, with exact chiral, flavour and spin symmetry for any arbitrary lattice spacing, appear to do better. Advocated as means to explore the large scale composition of QGP, various numerical investigations with staggered quarks found that except the pion (and the sigma) screening length, all others could be understood as multiples of the appropriate number of free quarks (or antiquarks). The pionic correlator on the other hand showed nontrivial structure, and the pionic screening length approached the ideal gas value only in the continuum limit. The simulations with overlap quarks yielded a pleasant surprise on both counts and the corresponding pion screening length was close to ideal gas value for even small temporal lattices.

Another fundamental aspect of QCD is the existence and location of the critical point in the $T-\mu_{B}$ plane, where $\mu_{B}$ is the chemical potential for baryon number. Based on symmetries and a variety models, the QCD phase diagram is expected to have a critical point for two light and one moderately heavy quark. Again chiral symmetry plays a crucial role in this : the transition at $\mu_{B}=0$ should be second order for two massless quarks, which turns into a cross over for light quarks. A line of first order phase transitions at finite density ought to terminate in a critical point.

\section{Ginsparg-Wilson Relation and $\mu \neq 0$.}

Exact chiral invariance for a lattice Fermion operator $D$ is assured if it satisfies the GinspargWilson relation [2] : $\left\{\gamma_{5}, D\right\}=a D \gamma_{5} D$. In particular, the chiral transformations [3] $\delta \psi=\alpha \gamma_{5}(1-$ 
$\left.\frac{a}{2} D\right) \psi$ and $\delta \bar{\psi}=\alpha \bar{\psi}\left(1-\frac{a}{2} D\right) \gamma_{5}$, leave the action $S=\sum_{x, y} \bar{\psi}(x) D_{x, y} \psi(y)$ invariant:

$$
\delta S=\alpha \sum_{x, y} \bar{\psi}_{x}\left[\gamma_{5} D+D \gamma_{5}-\frac{a}{2} D \gamma_{5} D-\frac{a}{2} D \gamma_{5} D\right]_{x y} \psi_{y}=0
$$

The Overlap Fermions, and the Domain Wall Fermions in the limit of large fifth dimension satisfy this relation. Thus these Fermions with exact chiral invariance on the lattice are ideal for studies of the QCD phase diagram. One needs to introduce chemical potential in their known actions to do so. For the staggered quarks, this was done by first finding an expression for the conserved number, $N$, on the lattice, and then adding $\mu N$ term to the action. It turned out [4] that this lead to $\mu^{2}$-dependent divergences in the continuum limit even for the free case, which were removed by further modification of the action. Since the non-locality of the Overlap or Domain Wall Fermions makes the construction of a conserved charge difficult, it was proposed [5] that the Wilson Dirac operator $D_{W}$ in the actions for these Fermions be modified using the same prescription as in the staggered case. This amounted to multiplying the link variable in the positive (negative) time direction by $K(a \mu)=\exp (a \mu)(L(a \mu)=\exp (-a \mu))$. As the $\gamma_{5} D_{W}(a \mu)$ is no longer Hermitian, the $D_{o v}=1+\gamma_{5} \operatorname{sgn}\left(\gamma_{5} D_{W}\right)$ definition of the overlap operator necessitated an extension of the sign function: For complex $\lambda=(x+i y)$ eigenvalue, $\operatorname{sgn}(\lambda)=\operatorname{sgn}(x)$. Using this operator, it was shown [6] showed numerically that no $\mu^{2}$-divergences exist in the free case $(U=1)$. We demonstrated [7] the absence of the divergence in the free case analytically for all $K, L$ such that $K(a \mu) \cdot L(a \mu)=1$.

We also claim [7] though that the chiral invariance is lost for nonzero $\mu$. This is easy to see by varying the quark fields again by the same infinitesimal chiral transformation as above :

$$
\delta S(a \mu)=\alpha \sum_{x, y} \bar{\psi}_{x}\left[\gamma_{5} D(a \mu)+D(a \mu) \gamma_{5}-\frac{a}{2} D(0) \gamma_{5} D(a \mu)-\frac{a}{2} D(a \mu) \gamma_{5} D(0)\right]_{x y} \psi_{y},
$$

whereas the extended sign function definition of the Dirac operator merely ensures

$$
\gamma_{5} D(a \mu)+D(a \mu) \gamma_{5}-a D(a \mu) \gamma_{5} D(a \mu)=0 .
$$

This is clearly not sufficient to make $\delta S=0$. This is true for both Overlap and Domain Wall Fermions and for any $K, L$.

A direct consequence is that the much desired exact chiral symmetry on lattice is lost for any $\mu \neq 0$, real or imaginary. Thus a $\mu$-dependent mass will be acquired by even massless quarks in the interacting theory. The behaviour of chiral condensate as a function of $\mu$ will therefore be necessarily smoothened, wiping out any chiral transition that may be present. Of course, depending on how strong the transition is, it will begin to show up for small enough $a$ or large enough $N_{T}$. How large a computational effort that may mean is a priori not clear. Recall that in the Taylor expansion method [8] to incorporate the effects of nonzero $\mu$, all the coefficients are evaluated at $\mu=0$. These will not suffer from any such lack of chiral invariance but the series in $\mu$ will be smooth, and will always exhibit convergence for any $\mu$.

One may be tempted to modify the chiral transformation itself for nonzero $\mu$ by demanding $\delta \psi=\alpha \gamma_{5}\left(1-\frac{a}{2} D(a \mu)\right) \psi$ and $\delta \bar{\psi}=\alpha \bar{\psi}\left(1-\frac{a}{2} D(a \mu)\right) \gamma_{5}$. Clearly, $\delta S(a \mu)=0$ in that case. This is, however, not permissible since $\gamma_{5} D(a \mu)$, i.e, the generator of the transformation is not Hermitian. Moreover, a symmetry transformations should not depend on the "external" tunable parameter $\mu$. Recall that the chemical potential is introduced for charges $N_{i}$ with $\left[H, N_{i}\right]=0$. At least, the 
symmetry should therefore not change as $\mu$ does. The most damaging practical consequence of such a modification of the chiral transformation is that the restoration of chiral symmetry due to an increase in $\mu$ cannot be addressed at all. For $T \neq 0$ with $\mu=0$, the symmetry group remains the same at each $T$, allowing a change in the chiral order parameter $\langle\bar{\psi} \psi\rangle(a m=0, T)$ to be interpreted as a change in the vacuum, i.e, restoration of the dynamically broken symmetry. With $\mu$-dependent chiral transformations, the symmetry groups are different at each $\mu$, with no obvious relation between the respective chiral condensates. It thus appears much more reasonable not to alter the transformations but to look for a better way to include the chemical potential in the Overlap Dirac operator.

\section{Free Overlap and Domain Wall Fermions: Analytical Results.}

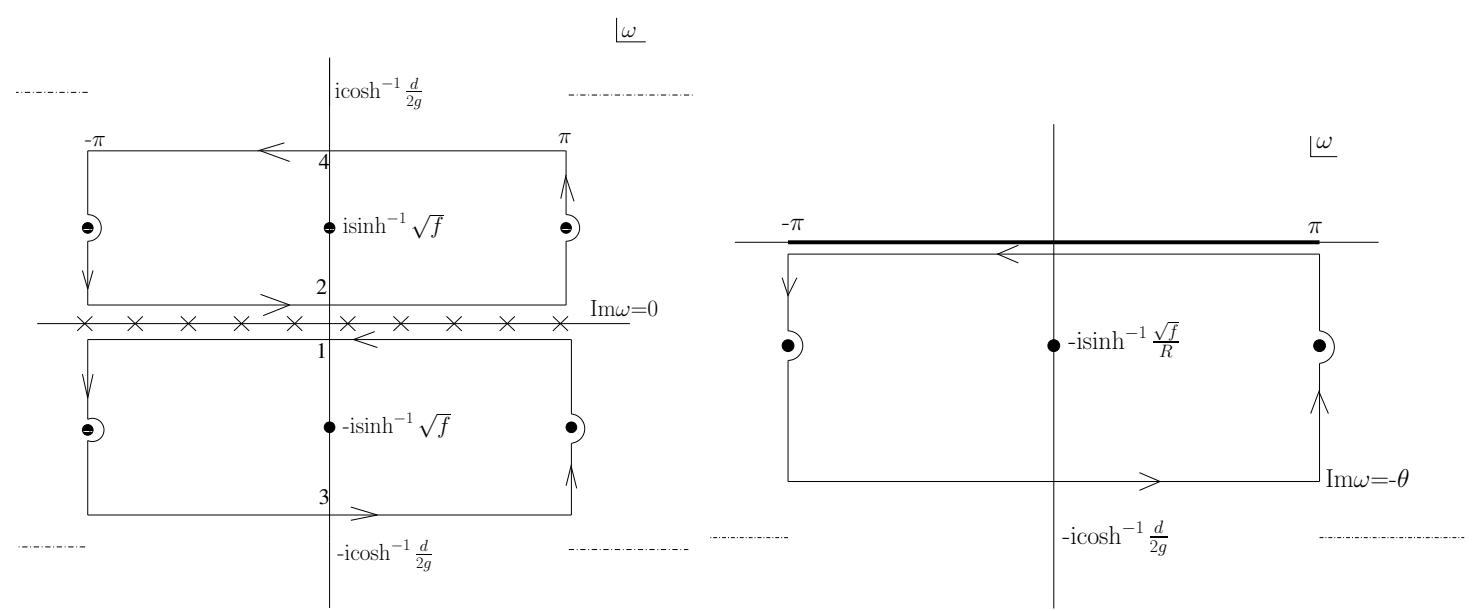

Figure 1: Contours in the complex $\omega$-plane for $\mu=0$ (left) and $T=0$ (right) cases.

We investigated thermodynamics of free overlap and domain wall Fermions both analytically and numerically to demonstrate that i) the negative mass parameter $0<M<2$ is irrelevant in the continuum limit, and ii) the absence of $\mu^{2}$-divergences for general $K$ and $L$. Using our numerical results, we also obtained an optimal range for $M$ in order to obtain small deviations from the continuum limit on coarse lattices.

The energy density, pressure, quark number susceptibility etc. can be obtained from $\ln \mathscr{Z}=$ ln det $D_{o v}$ by taking $T, V$, and $\mu$ or equivalently $a_{4}$ and $a$ and $a \mu$, partial derivatives. Here $V=N_{s}^{3} a^{3}$ and $T=1 /\left(N_{T} a_{4}\right)$. Since the Dirac operator is diagonal in momentum space, its eigenvalues can be used to compute $\mathscr{Z}$ :

$$
\begin{aligned}
\lambda_{ \pm} & =1-\left[\operatorname{sgn}\left(\sqrt{h^{2}+h_{5}^{2}}\right) h_{5} \pm i \sqrt{h^{2}}\right] / \sqrt{h^{2}+h_{5}^{2}}, \text { with } \\
h^{2}=\sum_{i=1}^{4} h_{i}^{2}, h_{j} & =-\sin a p_{j}, j=1,2 \text { and } 3, h_{4}=-a \sin \left(a_{4} p_{4}\right) / a_{4} \\
\text { and } h_{5} & =M-\sum_{j}^{3}\left[1-\cos \left(a p_{j}\right)\right]-a\left[1-\cos \left(a_{4} p_{4}\right)\right] / a_{4} .
\end{aligned}
$$

It is straightforward to show that $\varepsilon=3 P$ for all $a$ and $a_{4}$. Note that the free energy on finite volume is in general not equal to the pressure $|P|$, and thus will have $\mathrm{O}(1 / \mathrm{V})$ corrections spoiling 
the equality above. Hiding the spatial momentum $p_{j}$-dependence in terms of known functions [7] $g, d$ and $f$, the energy density on an $N_{s}^{3} \times N_{T}$ lattice is

$$
\begin{aligned}
\varepsilon a^{4}=\frac{2}{N_{s}^{3} N_{T}} \sum_{p_{j}, n} F\left(1, \omega_{n}\right) & =\frac{2}{N_{s}^{3} N_{T}} \sum_{p_{j}, n}\left[\left(g+\cos \omega_{n}\right)+\sqrt{d+2 g \cos \omega_{n}}\right] \\
& \times\left[\frac{\left(1-\cos \omega_{n}\right)}{d+2 g \cos \omega_{n}}+\frac{\sin ^{2} \omega_{n}\left(g+\cos \omega_{n}\right)}{\left(d+2 g \cos \omega_{n}\right)\left(f+\sin ^{2} \omega_{n}\right)}\right] .
\end{aligned}
$$

where $\omega_{n}\left(=a_{4} p_{4}\right)$ are the Matsubara frequencies. As in the case of the continuum, sum over $\omega_{n}$ can be carried out using the contour technique in the complex $\omega$-plane. The left panel in the Figure 1 displays the contour chosen for the $\mu=0$ case. The crosses denote the Matsubara frequencies, the circles denote the physical poles in eq. (3.3) corresponding to the zero of $\left(f+\sin ^{2} \omega\right)$ and the dashed lines correspond to the cuts. Evaluating the integrals, one obtains $\varepsilon a^{4}=4 N_{s}^{-3} \sum_{p_{j}}[\sqrt{f / 1+f}]\left[\exp \left(N_{T} \sinh ^{-1} \sqrt{f}\right)+1\right]^{-1}+\varepsilon_{3}+\varepsilon_{4}$, where $f=\sum_{j} \sin ^{2}\left(a p_{j}\right)$, and the contributions from the contours at the top and bottom are denoted by $\varepsilon_{3,4}$ respectively. In the continuum limit, the above expression reduces to the ideal gas energy density $\varepsilon_{S B}$ for all $M$, with the cuts moving away to infinity faster than the contours at the to and bottom.

For the case of $T=0$ but $\mu \neq 0$, the contour is displayed in the right panel of Figure 1. Essentially the same treatment goes through as above, if one defines $K(\mu)+L(\mu)=2 R \cosh \theta$ and $K(\mu)-L(\mu)=2 R \sinh \theta$ and substitutes in eq. (3.3) $R \sin \left(\omega_{n}-i \theta\right)$ for $\sin \omega_{n}$ and similarly for $\cos \omega_{n}$. After the contour integral one obtains

$\varepsilon a^{4}=\frac{1}{\pi N_{s}^{3}} \sum_{p_{j}}\left[2 \pi \operatorname{Res} F(R, \omega) \Theta(K(a \mu)-L(a \mu)-2 \sqrt{f})+\int_{-\pi}^{\pi} F(R, \omega) d \omega-\int_{-\pi}^{\pi} F(1, \omega) d \omega\right]$.

From the above expression, one notices that $R=K(a \mu) \cdot L(a \mu)=1$ ensures cancellation of the last two terms. For $R \neq 1$, expanding $R$ in powers of $\mu$, on the other hand, the $\mu^{2}$-divergence is explicitly seen in the continuum limit. Note the higher order terms in that case violate the Fermi surface condition in the $\Theta$-function and contribute for all $\mu$ on the lattice; they vanish in the continuum limit. From the $\Theta$-function in the expression above, it is also clear that $K$ and $L$ should be such that $K(a \mu)-L(a \mu)=2 a \mu+\mathscr{O}\left(a^{3}\right)$ in order that the usual Fermi surface condition is recovered. Furthermore, $K(0)=1=L(0)$ is necessary for consistency with the zero density Dirac operator. We have also shown that the above derivations go through for the Domain Wall Fermions [9] and for the general case of $T \neq 0$ and $\mu \neq 0$ for both the Overlap [7] and Domain wall Fermions.

\section{Free Overlap and Domain Wall Fermions: Numerical Results.}

Numerical evaluation of the physical observables is a simple evaluation of sums over all allowed momenta $a p_{j}=\left(2 \pi / N_{s}\right) n_{j}$ with $n_{j}=0, N_{s}-1$ and $a_{4} p_{4}=\left(2 n_{4}+1\right) \pi / N_{T}$ with $n_{4}=$ $0, N_{T}-1$. Fixing a large $\zeta=L T=N_{s} / N_{T}$ for thermodynamic limit, we let $N_{T} \rightarrow \infty$ to obtain the results in the continuum limit. Figure 2 shows $\Delta \varepsilon(\mu, T) / T^{4}=[\varepsilon(\mu, T)-\varepsilon(0, T)] / T^{4}$ for $\mu / T=0.5$ for the Overlap (top panel) and the Domain Wall (bottom) Fermions for $\zeta=4$. The $\zeta=5$ results have been checked to be indistinguishable from these. Both sets of results show small, $\sim 2-3 \%$, 

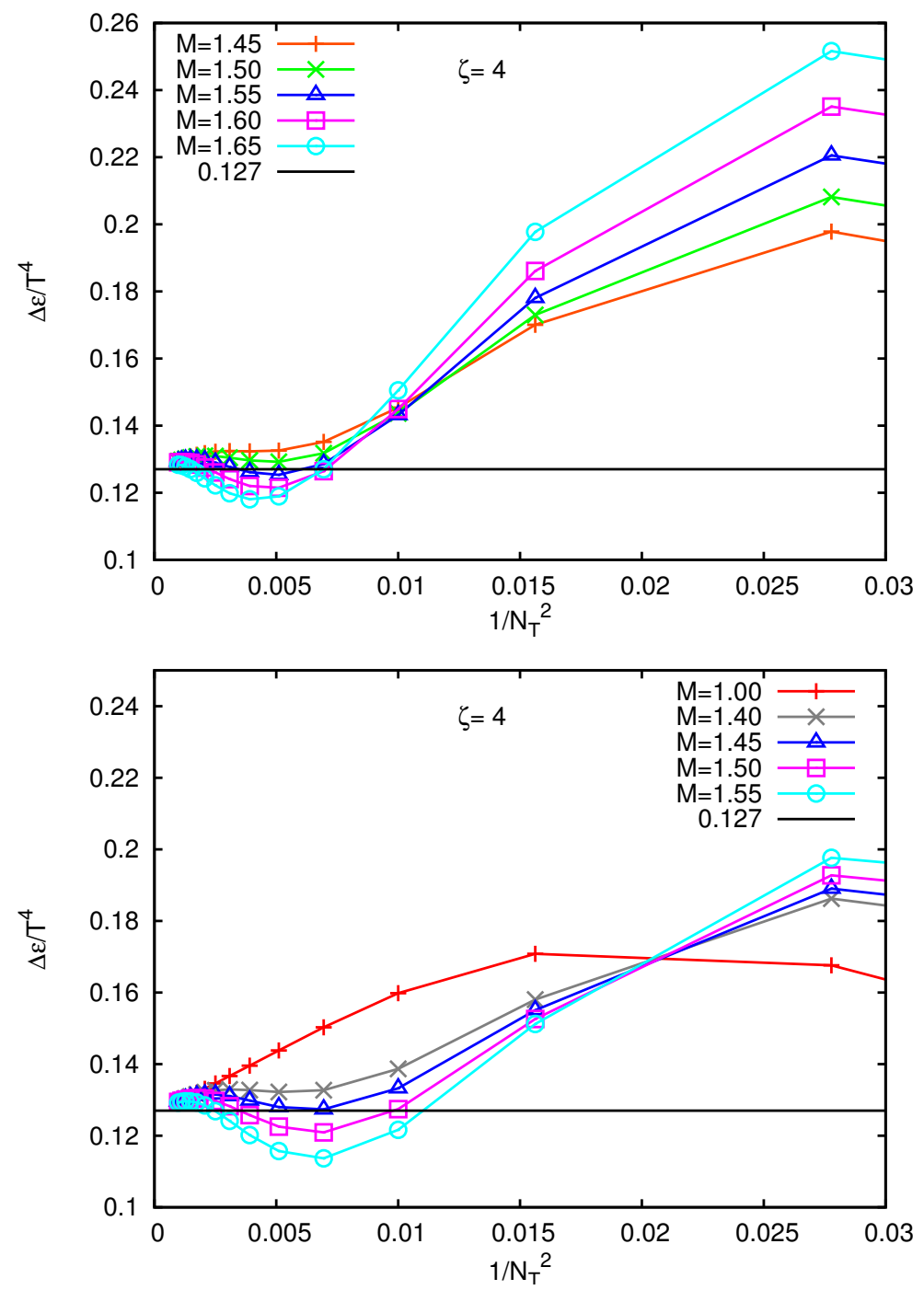

Figure 2: $\mu$-dependent contribution to the energy density for Overlap (top) and Domain Wall (bottom) Fermions for $\mu / T=0.5$.

deviations from the continuum result $(=0.127$ for $\mu / T=0.5)$ for $N_{T} \geq 12$ for a range of $M$, being $1.50 \leq M \leq 1.60$ for Overlap Fermions and $1.40 \leq M \leq 1.50$ for the Domain Wall Fermions. Note that the canonical $M=1$ choice yields a smoother $1 / N_{T}$ dependence but larger deviations.

Quark number susceptibility, defined as

$$
\chi=\frac{T}{V}\left(\frac{\partial^{2} \ln \operatorname{det} D}{\partial \mu^{2}}\right)_{T, V}
$$

can be worked out for the Overlap Fermions to be

$$
\chi=\frac{2 i}{N_{T} N_{s}^{3} a^{2}} \sum_{p_{j}, p_{4}}\left[\frac{-\left(h^{2} h_{4}+h_{4} h_{5} \cos \left(a p_{4}-i a \mu\right)\right) u}{s^{4}\left(s-h_{5}\right)^{2}}+\frac{v}{s^{2}\left(s-h_{5}\right)}\right] .
$$


The $\mathrm{u}$ and $\mathrm{v}$ in the expression above are

$$
\begin{aligned}
& u=2\left(s-h_{5}\right)\left(h_{4} \frac{\partial h_{4}}{\partial a \mu}+h_{5} \frac{\partial h_{5}}{\partial a \mu}\right)+s^{2}\left(\frac{\partial s}{\partial a \mu}-\frac{\partial h_{5}}{\partial a \mu}\right), \text { and } \\
& v=\frac{\partial h_{4}}{\partial a \mu}\left(2 h_{4}^{2}+h^{2}+h_{5} \cos \left(a p_{4}-i a \mu\right)\right)+h_{4} \frac{\partial h_{5}}{\partial a \mu} \cos \left(a p_{4}-i a \mu\right)+i h_{4} h_{5} \sin \left(a p_{4}-i a \mu\right),
\end{aligned}
$$

with $s^{2}=h^{2}+h_{5}^{2}$ and the $h_{i}$ as defined in eq.(3.1) but with $a_{4} p_{4} \rightarrow\left(a p_{4}-i a \mu\right)$. Similarly one can write down an expression for it for the Domain Wall Fermions. Figure 3 displays the results for the Overlap Fermions (top panel) and the Domain Wall Fermions (bottom panel) as a function of $1 / N_{T}^{2}$. The behaviour is each case is similar to the corresponding energy density case in Figure 2. In particular, the same range of $M$ seems to be optimal for both $\Delta \varepsilon$ and $\chi(\mu=0)$.
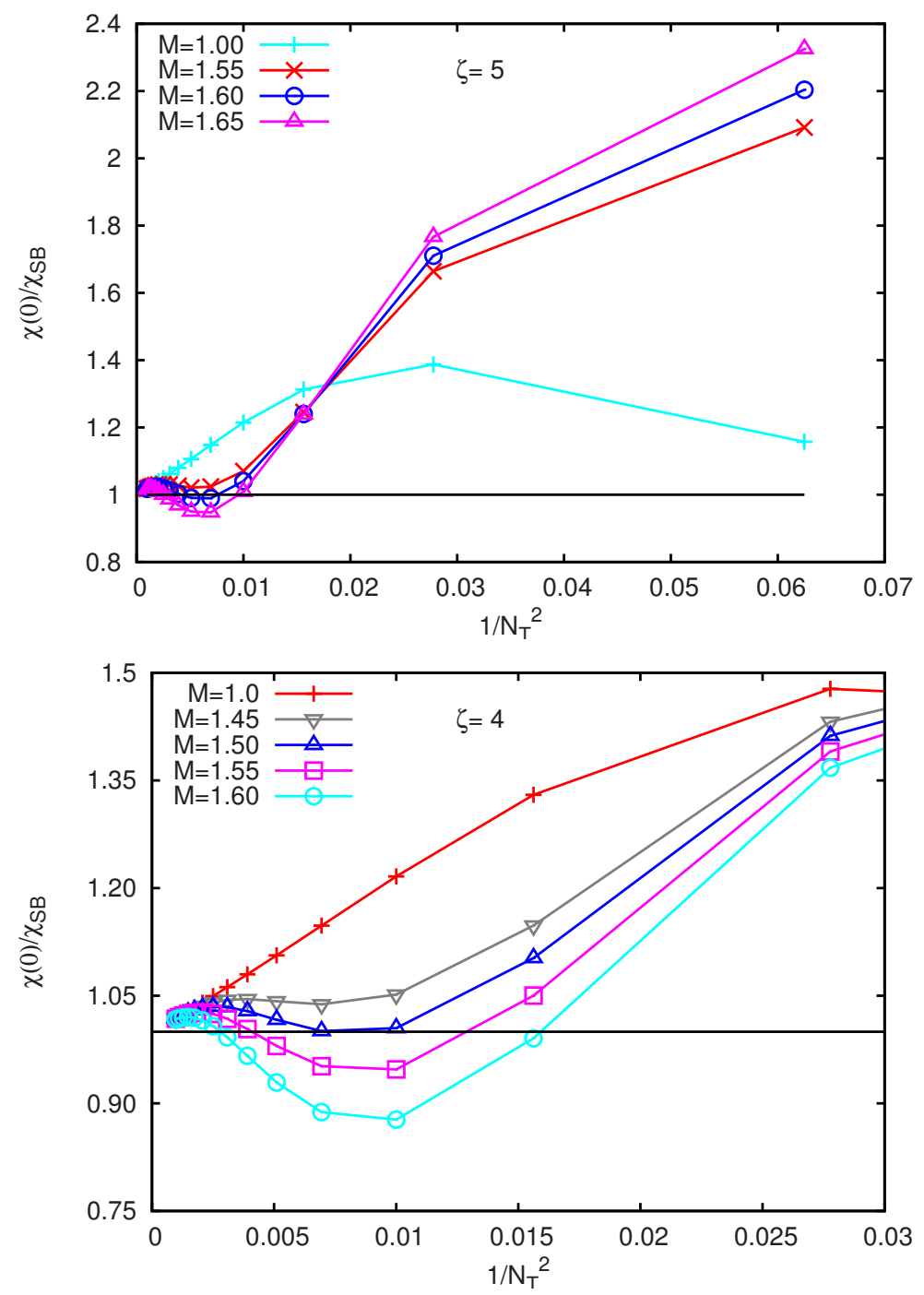

Figure 3: Quark number susceptibility as a function of $1 / N_{T}^{2}$ for Overlap (top) and Domain Wall (bottom) Fermions for $\mu=0$. 


\section{Summary.}

Exact chiral symmetry for the quark fields on the lattice, without any violation of other symmetries, such as the flavour or spin, is important for several investigations of QCD thermodynamics. Indeed, the existence of the critical point in the $T$ - $\mu_{B}$ phase diagram of QCD, and the nonperturbative determination of its location, depend on it. The currently popular choice of staggered Fermions may not be fully adequate for such studies. The Overlap and Domain wall Fermions are ideally suited but unfortunately lose their chiral invariance on introduction of chemical potential in the Bloch-Wettig method and its generalizations, as we showed above. We also proved analytically that no $\mu^{2}$-divergence exists in the continuum limit for both the Overlap and Domain Wall Fermions for the Bloch-Wetting method and an associated general class of functions $K(\mu)$ and $L(\mu)$ with $K(\mu) \cdot L(\mu)=1$. Our numerical results corroborate these findings, and lead us to an optimal range for the irrelevant parameter $M$. For the choice of $1.5 \leq M \leq 1.6(1.4 \leq M \leq 1.5)$, both the quark number susceptibility at $\mu=0$ and the $\mu$-dependent part of the energy density exhibited the smallest deviations from the ideal gas limit for $N_{T} \geq 12$ for Overlap (Domain Wall) Fermions.

\section{Acknowledgements.}

One of us (R.V.G.) wishes to thank the members of the local organizing committee for their kind hospitality which enabled his participation in the symposium.

\section{References}

[1] R. V. Gavai, S. Gupta and R. Lacaze, PoS LAT2006, 135 (2006); Phys. Rev. D78, 014502 (2008).

[2] P. H. Ginsparg and K. G. Wilson, Phys. Rev. D25, 2649 (1982).

[3] M. Luscher, Phys. Lett. B428, 342 (1998).

[4] N. Bilic and R. V. Gavai, Z. Phys. C23, 77 (1984); R. V. Gavai, Phys. Rev. D32, 519 (1985).

[5] J. Bloch and T. Wettig, Phys. Rev. Lett. 97, 012003 (2006); Phys. Rev. D76 114511 (2007).

[6] C. Gattringer and L. Liptak, Phys. Rev. D76, 054502 (2007).

[7] D. Banerjee, R. V. Gavai and S. Sharma, Phys. Rev. D78 014506 (2008).

[8] R. V. Gavai and S. Gupta, Phys. Rev. D68, 034506 (2003); Phys. Rev. D71, 114014 (2005).

[9] R. V. Gavai and S. Sharma, J. Phys. G 35, 104097 (2008), and in preparation. 